\title{
Bridge Treatments for HCC in the Waiting List for Liver Transplantation
}

\author{
Maurizio Pompili ${ }^{*}, 1$, Valeria Abbate ${ }^{1}$, Erica Nicolardi ${ }^{1}$, Francesca Romana Ponziani ${ }^{1}$, \\ Laura Riccardi ${ }^{1}$, Antonio Gasbarrini ${ }^{1}$, Antonio Grieco ${ }^{1}$, Alfonso W. Avolio ${ }^{2}$ and \\ Gian Ludovico Rapaccini ${ }^{1}$
}

\author{
${ }^{I}$ Department of Internal Medicine, Catholic University of Rome, Largo Agostino Gemelli, 8 - 00168, Roma, Italy \\ ${ }^{2}$ Department of Surgery, Catholic University of Rome, Largo Agostino Gemelli, 8-00168, Roma, Italy
}

\begin{abstract}
During the past years, several therapeutic procedures have been proposed as bridging treatments in patients with hepatocellular carcinoma waiting for liver transplantation. Resective surgery, transarterial chemoembolization, radiofrequency thermal ablation and percutaneous ethanol injection are the most experienced, with the aim to decrease the rate of drop-out from LT waiting list, and the risk of HCC recurrence after transplant. Indeed, for patients within the Milan criteria, a time on waiting list exceeding 6-12 months is a known risk factor of tumor progression and drop out. For this reason, the application of bridging treatments in these patients might be reasonable and several studies in recent years have documented their usefulness to control tumor progression before the transplant. However, the favourable impact of these treatments on post-transplant patients' survival is still under investigation and the available studies provide controversial results. Bridging therapies have also been used for the downstaging of tumors exceeding the conventional "Milan criteria". Some recent data regarding multimodal sequential therapies seem to report promising results in terms of overall and disease-free survival of treated patients attaining effective downstaging before transplant.
\end{abstract}

Keywords: Hepatocellular carcinoma, transarterial chemoembolization, percutaneous treatments, liver cirrhosis, liver transplantation.

\section{INTRODUCTION}

Hepatocellular carcinoma (HCC) emerging in liver cirrhosis within the so called Milan criteria (1 nodule smaller than $5 \mathrm{~cm}$ or no more than 3 nodules smaller than $3 \mathrm{~cm}$ ) is recognized everywhere as an indication to liver transplantation (LT). In this setting, the major problem is a high cumulative probability of drop-out from LT waiting list for tumor progression, which has been reported to range between $7 \%$ and $11 \%$ at 6 months, increasing to $38 \%$ at 12 months from the enrollment $[1,2]$.

The use of the Model for End Stage Liver Disease (MELD) has provided an important advantage in the management of liver transplantation waiting list and to $\mathrm{HCC}$ patients listed for LT, since 22 additional MELD points are given to patients with stage 2 tumors, according to TNM classification [3]. In spite of this, in the U.S.A. the cumulative 6-months probability to drop out from LT waiting list in the MELD era is comparable to that of patients without malignancy $(16.9 \%$ vs. $13.5 \%)$ but becomes significantly higher at 12 months (31.8 \% vs. 19.1\%); significant risk factors for drop out are a high MELD score or alpha-fetoprotein (AFP) serum levels, a large HCC size and an advanced patient's age [4].

For this reason, several therapeutic procedures have been proposed in the past as bridge treatments for $\mathrm{HCC}$ patients in

*Address correspondence to this author at the Department of Internal Medicine, Università Cattolica del Sacro Cuore, Largo A. Gemelli, 8 00168 Roma, Italy; Tel: + 003906 30154334; Fax: + 00390635502775 ; E-mail: mpompili@rm.unicatt.it
LT setting. The rationale for their use is the possible decrease of drop-out rate from the waiting list and of the evidence of HCC recurrence after transplantation, which is less than $15 \%$ in patients within Milan criteria undergoing LT without any tumor treatment [5]. These beneficial effects may also determine an improvement of post-LT overall survival. Furthermore, several Authors believe that Milan criteria could be expanded without affecting tumor recurrence after LT and patient's survival; the most common approach to these patients has been to decrease the size of the tumor mass within the conventional transplantability criteria [6].

In this article, we analyze the indications and the effectiveness of the various treatment modalities currently applied to HCC patients awaiting for LT to avoid the overcoming of Milan criteria while in the waiting list, and to downstage patients listed beyond the conventional transplantability criteria.

\section{RESECTIVE SURGERY}

Liver resection has been evaluated in the setting of LT with contrasting results. Even if, in most transplant centres, transarterial chemoembolization (TACE) and percutaneous ablation therapies are the preferred bridging therapeutic options, the theoretical advantage of surgery in this setting could be a better control of tumor growth since, often, TACE and percutaneous treatments do not achieve complete tumor necrosis. However, a surgical bridging treatment in patients listed for LT entails more periprocedural risks, can only be proposed to well compensated patients without severe portal 
hypertension, and can make the transplant procedure more difficult. In a paper by Adam et al., the overall survival and disease free survival rates of patients undergoing LT after HCC resection were significantly lower than in cirrhotic patients with $\mathrm{HCC}$ undergoing primary LT [7]. On the contrary, Belghiti et al. reported that postoperative course, complications, and 3- and 5-year survival rates did not differ significantly in HCC cirrhotic patients undergoing primary LT or secondary LT after resection [8]. Resection can also be considered in two different settings. First, surgery can be used as HCC primary therapy and LT reserved as rescue therapy for patients developing recurrence; in the series by Poon et al., about $80 \%$ of patients were still evaluable for salvage LT at the time of tumor recurrence [9]. Secondly, resection may be used to select patients by the analysis of the resected specimen, in the presence of an undifferentiated histotype, satellitosis, microvascular invasion or capsular effraction [10]. On the whole, according to Belghiti et al., resection and transplantation should be considered, in selected cases, as associated rather than as alternative procedures [11].

\section{TRANSARTERIAL CHEMOEMBOLIZATION}

TACE has been extensively used in the past. There are not published data from prospective randomized studies but, during the nineties, excellent results in terms of post-LT recurrence and patient survival have been reported $[12,13]$.

Lesurtel et al. published an interesting paper on the usefulness of TACE in HCC patients undergoing LT, according to the criteria of the evidence based medicine. The first question was: does TACE impact on waiting list dropout? Authors concluded that there was no sufficient evidence to provide a response due to the absence of prospective comparative studies [14]. Hayashi et al. reported a discouraging $35 \%$ of drop out rate in patients with stage 1 or $2 \mathrm{HCC}$ and with a mean waiting time of 340 days treated with TACE [15]. However, an analysis of the most recent series including patients treated with TACE or transarterial chemoinfusion before LT, showed that the drop out rate due to tumor progression is low, ranging between $2.9 \%$ and $13 \%$ with a mean waiting time in the transplant list exceeding 6 months (Table 1) [16-19].

Table 1. Drop out Rate due to Tumor Progression in HCC Patients Waiting for Liver Transplantation (LT) and Treated by Transarterial Chemoembolization (TACE) or Transarterial Chemoinfusion

\begin{tabular}{|c|c|c|c|}
\hline Author & $\begin{array}{c}\text { Number of } \\
\text { patients }\end{array}$ & $\begin{array}{c}\text { Mean waiting } \\
\text { (days) }\end{array}$ & $\begin{array}{c}\text { Drop-out (\%) (HCC } \\
\text { progression) }\end{array}$ \\
\hline \hline $\begin{array}{c}\text { Millonig G } \\
2007\end{array}$ & 68 & 274 & $2 / 68(2.9 \%)$ \\
\hline Majno P 2007 & 43 & 203 & $4 / 43(10 \%)$ \\
\hline Alba E 2008 & 56 & 201 & $7 / 63(11 \%)$ \\
\hline $\begin{array}{c}\text { De Luna } \\
\text { 2008 }\end{array}$ & 95 & 342 & $12 / 95(13 \%)$ \\
\hline
\end{tabular}

The second important problem debated in the already cited paper was the impact of preoperative TACE on HCC patients' survival after LT. Again, the conclusion was that there was no sufficient data from prospective randomized studies to be evaluated [14]. A recent multicentric retrospective case-control study, compares $100 \mathrm{HCC}$ patients treated with TACE before transplantation and $100 \mathrm{HCC}$ patients transplanted without any bridge treatment. Both 5year survival (59\% in treated and not treated patients) and 5year disease free survival (69\% vs. 64\% respectively) did not differ between the two groups. At post-explant analysis, a total or subtotal tumor necrosis exceeding $80 \%$ was found in about $30 \%$ of treated patients, but with no difference in 5year survival in respect to untreated patients $(63 \%$ vs. 54\%) [20]. In the largest available series, the rate of patients treated by TACE reaching a complete tumor necrosis is quite uniform, ranging between $27 \%$ and $55 \%$ in patients within Milan criteria (Table 2) [16-21]. Reasonably, these could be the patients who could receive a significant survival benefit from TACE before LT by reducing the risk of $\mathrm{HCC}$ recurrence after LT. This seems to be confirmed by Millonig et al., in a series of $116 \mathrm{HCC}$ patients undergoing TACE before transplantation. Most of the patients were within Milan criteria and complete tumor necrosis was obtained in $27 \%$ of the cases. Five-year survival rate was higher in patients with a complete tumor necrosis compared to those with partial necrosis $(86 \%$ vs. $66 \%)$ even if this difference did not reach statistical significance [16].

Table 2. Performance of TACE Performed before Liver Transplantation (LT) in Terms of Total-Subtotal, Partial or Inadequate Necrosis of the Treated Hepatocellular Carcinoma (HCC). MC: Milan Criteria

\begin{tabular}{|c|c|c|c|c|}
\hline \multicolumn{5}{|c|}{ Tumor necrosis at explant analysis } \\
\hline Author & $\begin{array}{c}\text { Number } \\
\text { of HCCs }\end{array}$ & $\begin{array}{c}\text { Total-Subtotal } \\
(\mathbf{8 0 - 1 0 0 \% )}\end{array}$ & $\begin{array}{c}\text { Partial } \\
(<\mathbf{8 0} \%)\end{array}$ & $\begin{array}{c}\text { Inadequate } \\
(<\mathbf{5 0} \%)\end{array}$ \\
\hline \hline $\begin{array}{c}\text { Majino P } \\
1997\end{array}$ & $(54 \mathrm{pts})$ & $27 \%$ & $27 \%$ & $46 \%$ \\
\hline $\begin{array}{c}\text { Graziadei I } \\
2003\end{array}$ & $(63$ pts $)$ & $\begin{array}{c}\text { In MC 29\% } \\
\text { Out MC 0\% }\end{array}$ & $\begin{array}{c}67 \% \\
73 \%\end{array}$ & $\begin{array}{c}4 \% \\
27 \%\end{array}$ \\
\hline $\begin{array}{c}\text { Decaens T } \\
2005\end{array}$ & 100 & $30 \%$ & $20 \%$ & $50 \%$ \\
\hline $\begin{array}{c}\text { Millonig G } \\
2007\end{array}$ & $(106 \mathrm{pts})$ & $27 \%$ & $77 \%$ & \\
\hline Alba E 2008 & 128 & $38 \%$ & $62 \%$ & $48 \%$ \\
\hline $\begin{array}{c}\text { De Luna W } \\
2009\end{array}$ & 122 & $\begin{array}{c}\text { In MC 55\% } \\
\text { Out MC 33\% }\end{array}$ & $\begin{array}{c}18 \% \\
33 \%\end{array}$ & $\begin{array}{c}27 \% \\
33 \%\end{array}$ \\
\hline
\end{tabular}

Another important question, is the safety of TACE in patients waiting for LT. Since local arteritis may complicate TACE, recipients could be exposed to an increased incidence of complications, such as hepatic artery thrombosis. However, this hypothesis has not been confirmed [17, 22, 23].

\section{RADIOFREQUENCY THERMAL ABLATION}

Radiofrequency thermal ablation (RFA) has gained wide diffusion during the last years as an effective procedure for small HCC not amenable to surgical resection. Thermal 
ablation may be performed using cool or hook needles with comparable results [24]. Several papers analyzed the usefulness of RFA as a bridging treatment to LT in HCC patients. However, only two studies provided data about the effectiveness of RFA in avoiding patients' drop out from LT waiting list due to tumor progression. The rate has been found to be $0 \%$ in one study [25] and $12 \%$ in another one [26].

Some papers about the efficacy of RFA as bridge to transplant in HCC patients have been published during the last years. These studies show that complete tumor necrosis (evaluated on the explanted liver) may be achieved in 47$75 \%$ of the cases with a mean value of $58 \%$ [25-29]. A clear different effectiveness can be demonstrated analysing tumors of different size. In fact, the rate of complete necrosis ranges between $50 \%$ and $78 \%$ in tumors up to $3 \mathrm{~cm}$ and between $13 \%$ and $43 \%$ in larger tumors [25-27, 29]. Furthermore, a tumor size exceeding $3 \mathrm{~cm}$ resulted the only risk factor for HCC persistence after treatment in two studies [25, 27].

As regards RFA-related complications in HCC patients listed for LT, the analysis of the largest available series demonstrates that the procedure is quite safe. Indeed, the mean rate of major complications was as high as $7 \%$, including one case of death due to peritoneal bleeding, two cases of acute peritonitis/cholecystitis, and one case each of severe liver failure treated by urgent transplantation, severe persistent liver failure, biliary stenosis, arterial hemorrage and small bowel perforation [25-28]. Additionally, the risk of tumor seeding along the needle track through the abdominal wall appears to be low since only one case has been reported in literature [30]; HCC recurrence after LT has been shown to be rare (3\% on average) (Table 3$)$.

Table 3. HCC Recurrence after Liver Transplantation (LT) in Patients Undergoing Radiofrequency Thermal Ablation (RFA) during the Waiting Time

\begin{tabular}{|c|c|c|c|}
\hline Author/Year & Patients & $\begin{array}{c}\text { Follow Up (Months, } \\
\text { Mean and Range) }\end{array}$ & $\begin{array}{c}\text { Recurrence } \\
\text { (Patients) }\end{array}$ \\
\hline \hline $\begin{array}{c}\text { Mazzaferro } \\
2004\end{array}$ & 50 & 22 & 2 \\
\hline Lu 2005 & 41 & $15(0-55)$ & 0 \\
\hline Pompili 2005 & 30 & $34(3-132)$ & 0 \\
\hline Brillet 2006 & 16 & $25(0-40)$ & 1 \\
\hline Rodr.Sanj. 2008 & 28 & $32(2-78)$ & 2 \\
\hline \multicolumn{3}{|c|}{ Overall recurrence: $5 / 165(3.0 \%)$} \\
\hline
\end{tabular}

\section{OTHER TREATMENTS}

TACE and RFA are the bridge treatments to LT most frequently used in HCC patients, but also other therapeutic options have been proposed. Percutaneous ethanol injection (PEI) is the oldest and most experienced locoregional treatment for HCC, but is rarely used as a bridge to LT. In the largest series by Castrogaudin et al., ethanol injection provided a complete necrosis in $58 \%$ of patients with small tumors of less than $3 \mathrm{~cm}$ [31], while in our multicentric survey, the rate of complete necrosis was of $30 \%$, in tumors of the same size [27]. Both studies reported no PEI-related major complications and no case of total necrosis in tumors larger than $3 \mathrm{~cm}[27,31]$.

Percutaneous laser ablation (PLA) has been recently shown to be an effective technique to obtain HCC thermal ablation in patients ineligible for surgical resection $[32,33]$. We recently reported that PLA could provide results comparable to RFA in HCC patients waiting LT, since the rate of complete necrosis found at the post-explant analysis in nodules up to $3 \mathrm{~cm}$ was of $62 \%$ [34]. Possible advantages of PLA include the feasibility of the treatment in patients with severe clotting impairment precluding RFA, and the lower overall cost of the procedure.

The efficacy of radioembolization with 90Yttrium microspheres has been recently evaluated by Riaz et al. [35] on 38 nodules in 35 patients, 33 transplanted; a complete necrosis was observed in $89 \%$ of lesions up to $3 \mathrm{~cm}$ and in $65 \%$ of lesions between 3 and $5 \mathrm{~cm}$.

\section{DOWNSTAGING OF HCC BEYOND MILAN CRITERIA BEFORE LT}

It is well known that an acceptable post-LT survival may be also achieved expanding the limits of tumor size admitted for LT using the so called "UCSF criteria" (single tumor $\leq$ $6.5 \mathrm{~cm}$, or $\leq 3$ tumors with the largest being $\leq 4.5 \mathrm{~cm}$ and a total tumor burden $\leq 8 \mathrm{~cm}$ ) [36]. On the other hand, downstaging of HCC within the Milan criteria could be an attractive alternative; theoretically, this process allows the selection of tumors with a more favourable biology, which may be more likely to respond to down-staging treatments and to have a good outcome after LT. At this regard, the largest series has been reported by Yao et al. [6]; 61 HCC patients beyond $\mathrm{T} 2$ stage were included in a liver transplant program and treated with a multimodal downstaging including TACE, RFA, PEI and resective surgery. Eighteen patients dropped out for tumor progression and the only significant negative prognostic factor was AFP serum values $>1000 \mathrm{ng} / \mathrm{ml}$. 43 patients were downstaged within the Milan criteria according to imaging and observed for 3 months before listing; finally, 35 of these patients were transplanted. The post-explant analysis confirmed, for most of them, a tumor within T2 stage and, during the follow up, there was no evidence of recurrence, with the intention to treat survival of $88 \%$ at 1 year and of $69 \%$ at 5 years.

Some other recent papers provided encouraging results too. In a study by Chapman et al., 76 patients with HCC stage 3 or $4 \mathrm{a}$ were tentatively downstaged to T2 tumor using TACE. 18 patients (24\%) achieved a complete response and 17 of them were transplanted; the 5-year survival rate and HCC recurrence rate after LT were of $94 \%$ and $6 \%$, respectively [37]. De Luna et al. included 27 patients with HCC beyond Milan criteria in a downstaging program performed with TACE. 17 patients were considered downstaged to T2 tumor $(63 \%)$ and 15 underwent LT, with a 3-year survival rate of $79 \%$ and a $\mathrm{HCC}$ recurrence rate of 7\% [19]. Another important study was performed at the University of Bologna in Italy, including $177 \mathrm{HCC}$ patients evaluated for LT with a single tumor $\leq 8 \mathrm{~cm}$ or a bifocal tumor $\leq 5 \mathrm{~cm}$ or a multifocal tumor with less than 6 nodules, each smaller than $4 \mathrm{~cm}$ with a total diameter $\leq 12 \mathrm{~cm} .48$ patients were beyond 
the Milan criteria and underwent an adjuvant multimodal therapy using TACE, RFA, PEI and resective surgery. Once downstaged to T2 with AFP $<400 \mathrm{ng} / \mathrm{ml}$ and a stability of the disease for 3 months, they were listed for LT. Thirty-two patients were transplanted $(67 \%)$ with a drop-out rate of $27 \%$, a 5 -year intention to treat survival of $56 \%$ and a 3 -year disease free survival of $71 \%$ [38].

\section{IMPACT ON POST-LT SURVIVAL OF HCC BRIDGE TREATMENTS}

Independently from the treatment, a key question remains to be answered: do bridge treatments improve survival in HCC patients submitted to LT? Apart from monocentric retrospective series showing contradictory results,prospective randomized studies are lacking. In a study by Bharat et al., $46 \mathrm{HCC}$ patients undergoing various bridge treatments before LT were compared to 46 matched HCC patients transplanted without any treatment. The 5-year survival was significantly higher in the treated group $(82 \% v s .52 \%)$ and the gain in survival was evident only for patients with tumor stage T2-T4, but not for patients with tumor T0-T1. Even the 5 -year disease free survival was higher in the treated group (84\% vs. $76 \%)$ but this difference was not statistically significant [39]. In a recent study by Lao et al., 91 untreated HCC patients submitted to LT were compared to 33 patients with HCC undergoing TACE, RFA or ethanol injection before LT; treated patients had significantly larger tumors. HCC recurred only in 9 untreated patients and the factors significantly linked to tumor recurrence were MELD score < 14 , AFP $>1000 \mathrm{ng} / \mathrm{ml}$ and the absence of bridge treatment before transplant. The observed disease free survival was better in treated patients, but the cumulative survival was not significantly different [40]. Heckmann et al. compared the outcomes of $50 \mathrm{HCC}$ patients treated with a bridge therapy with $73 \mathrm{HCC}$ patients not treated before LT; they found no significant trend towards the improvement of 5-year survival in treated patients $(81 \% v s .71 \%)$ [41]. Finally, the study by Porrett et al., comparing 30 treated to 33 untreated patients before transplant, failed to show any survival difference between the groups. However, it should be pointed out that only $20 \%$ of the treated patients had a complete $\mathrm{HCC}$ necrosis at the post-explant analysis [30].

\section{CONCLUSION}

Due to the absence of prospective randomized studies, there is no data providing level I evidence that to treat HCC can reduce the drop out rate from the waiting list due to tumor progression and improve post-LT survival [42]. On the other side, for patients listed within the Milan criteria, the prolongation of the waiting time over 6-12 months without treatment is a well recognized risk factor of tumor progression and drop out from the list or post-transplant tumor recurrence $[1,2,4]$. For this reason, the best strategy for this subgroup of patients should be to perform the transplant within 6 months, avoiding the pre-transplant therapy [11]. However, in agreement with the current AASLD guidelines for the management of $\mathrm{HCC}$, if a prolonged waiting time is predicted, the application of bridging treatments appears to be reasonable in order to contain tumor progression [43].
TACE should be reserved to lesions $>3 \mathrm{~cm}$, eventually in multimodal treatment strategies or applied to all cases not eligible to percutaneous treatments, independently from tumor size. One third of the cases achieve a complete necrosis of the treated lesion, which could lead to improved survival after LT. RFA could be the treatment of first choice for lesions up to $3 \mathrm{~cm}$, since a complete tumor necrosis could be obtained in more than $60 \%$ of the cases at the postexplant analysis. The risk of major complications related to RFA in these patients appears to be quite low, but it should be a good clinical practice to limit the needle insertions and to avoid the treatment of superficially located lesions. PEI seems to show a lower efficacy, and should be reserved to small lesions located in sites considered "dangerous" for RFA (e.g. near the gallbladder or bowel loops). PLA and radioembolization with 90 Yttrium microspheres appear to be promising therapeutic tools, but further studies are needed to confirm their role and efficacy in these patients. Anyhow, all the ablation procedures should be evaluated with caution in patients with a reduced liver function, in order to avoid irreversible liver failure and severe complications precluding LT.

In carefully selected patients, HCC downstaging using TACE alone or combined in multimodal sequential therapies to meet the conventional criteria for LT, seems to provide promising results in terms of overall and disease-free survival. Furthermore, the achievement of T2 stage on imaging methods, a stable disease after effective downstaging during a 3-months follow up period, and AFP serum value $<1000$ $\mathrm{ng} / \mathrm{ml}$ before and $<400 \mathrm{ng} / \mathrm{ml}$ after a successful downstaging, seem to be predictive of favourable post-LT outcome in downstaged patients $[6,38]$.
ABBREVIATIONS
$\mathrm{HCC}=$ Hepatocellular carcinoma
LT $=$ Liver transplantation
MELD $=$ Model for end stage liver disease
AFP $=$ Alpha-fetoprotein
TACE $=$ Transarterial chemoembolization
RFA $=$ Radiofrequency thermal ablation
PEI = Percutaneous ethanol injection
PLA $=$ Percutaneous laser ablation
$\mathrm{UCSF}=$ University of California, San Francisco
AASLD $=$ American Association for the Study of the Liver

\section{REFERENCES}

[1] Llovet JM, Fuster J, Bruix J. Intention-to-treat analysis of surgical treatment for early hepatocellular carcinoma: resection versus transplantation. Hepatology 1999; 39: 1434-40.

[2] Yao FY, Bass NM, Nikolai B, et al. Liver transplantation for hepatocellular carcinoma: analysis of survival according to the intention-to-treat principle and dropout from the waiting list. Liver Transpl 2002; 8: 873-83. 
[3] Roayaie K, Feng S. Allocation policy for hepatocellular carcinoma in the MELD era: room for improvement? Liver Transpl 2007; 13 (Suppl 2): S36-43.

[4] Freeman RB, Edwards EB, Harper AM. Waiting list removal rates among patients with chronic and malignant liver diseases. Am J Transplant 2006; 6: 1416-21.

[5] Llovet JM, Burroughs A, Bruix J. Hepatocellular carcinoma. Lancet 2003; 362: 1907-17.

[6] Yao FY, Kerlan RK Jr, Hirose R, et al. Excellent outcome following down-staging of hepatocellular carcinoma prior to liver transplantation: an intention-to-treat analysis. Hepatology 2008; 48: 819-27.

[7] Adam R, Azoulay D, Castaing D, et al. Liver resection as a bridge to transplantation for hepatocellular carcinoma on cirrhosis: a reasonable strategy? Ann Surg 2003; 238: 508-18.

[8] Belghiti J, Cortes A, Abdalla EK, et al. Resection prior to liver transplantation for hepatocellular carcinoma. Ann Surg 2003; 238: 885-92.

[9] Poon RT, Fan ST, Lo CM, Liu CL, Wong J. Long-term survival and pattern of recurrence after resection of small hepatocellular carcinoma in patients with preserved liver function: implications for a strategy of salvage transplantation. Ann Surg 2002; 235: $373-$ 82.

[10] Sala M, Fuster J, Llovet JM, et al. High pathological risk of recurrence after surgical resection for hepatocellular carcinoma: an indication for salvage liver transplantation. Liver Transpl 2004; 10 : 294-300.

[11] Belghiti J, Carr BI, Greig PD, Lencioni R, Poon RT. Treatment before liver transplantation for HCC. Ann Surg Oncol 2008; 15: 993-1000.

[12] Harnois DM, Steers J, Andrews JC, et al. Preoperative hepatic artery chemoembolization followed by orthotopic liver transplantation for hepatocellular carcinoma. Liver Transpl Surg 1999; 5: 192-9.

[13] Spreafico C, Marchianò A, Regalia E, et al. Chemoembolization of hepatocellular carcinoma in patients who undergo liver transplantation. Radiology 1994; 192: 687-90.

[14] Lesurtel M, Mullhaupt B, Pestalozzi BC, Pfammatter T, Clavien PA. Transarterial chemoembolization as a bridge to liver transplanation for hepatocellular carcinoma: an evidence-based analysis. Am J Transpl 2006; 6: 2644-50.

[15] Hayashi PH, Ludkowski M, Forman LM, et al. Hepatic artery chemoembolization for hepatocellular carcinoma in patients listed for liver transplantation. Am J Transplant 2004; 4: 782-7.

[16] Milllonig G, Graziadei IW, Freund MC, et al. Response to preoperative chemoembolization corrrelates with outcome after liver transplantation in patients with hepatocellular carcinoma. Liver Transpl 2007; 13: 272-9.

[17] Majno P, Giostra E, Mentha G. Management of hepatocellular carcinoma on the waiting list before liver transplantation: time for controlled trials? Liver Transpl 2007; 13: S27-35.

[18] De Luna W, Sze DY, Ahmed A, et al. Transarterial chemoinfusion for hepatocellular carcinoma as downstaging therapy and a bridge toward liver transplantation.Am J Transplant 2000; 9: 1158-68.

[19] Alba E, Valls C, Dominguez J, et al. Transcatheter arterial chemoembolization in patients with hepatocellular carcinoma on the waiting list for orthotopic liver transplantation. AJR Am J Roentgenol 2008; 190: 1341-8.

[20] Decaens T, Roudot-Thoraval F, Bresson-Hadni S, et al. Impact of pretransplantation transarterial chemoembolization on survival and recurrence after liver transplantation for hepatocellular carcinoma. Liver Transpl 2005; 11: 767-75.

[21] Graziadei IW, Sandmueller $\mathrm{H}$, Waldenberger $\mathrm{P}$, et al. Chemoembolization followed by liver transplantation for hepatocellular carcinoma impedes tumor progression while on the waiting list and leads to excellent outcome. Liver Transpl 2003; 9: 557-63.

[22] Richard HM, Silberzweig JE, Mitty HA, Lou WY, Ahn J, Cooper JM. Hepatic arterial complications in liver transplant recipients treated with pretransplantation chemoembolization for hepatocellular carcinoma. Radiology 2000; 214: 775-9.
[23] Pérez Saborido B, Meneu JC, Moreno E, García I, Moreno A, Fundora Y. Is transarterial chemoembolization necessary before liver transplantation for hepatocellular carcinoma? Am J Surg 2005; 190: 383-7.

[24] Shibata T, Shibata T, Maetani Y, Isoda H, Hiraoka M. Radiofrequency ablation for small hepatocellular carcinoma: prospective comparison of internally cooled electrode and expandable electrode. Radiology 2006; 238: 346-53.

[25] Mazzaferro V, Battiston C, Perrone S, et al. Radiofrequency ablation of small hepatocellular carcinoma in cirrhotics awaiting liver transplantation. A prospective study. Ann Surg 2004; 240: 900-9.

[26] Lu DS, Yu NC, Raman SS, et al. Percutaneous radiofrequency ablation of hepatocellular carcinoma as a bridge to liver transplantation. Hepatology 2005; 41: 1130-7.

[27] Pompili M, Mirante VG, Rondinara G, et al. Percutaneous ablation procedures in cirrhotic patients with hepatocellular carcinoma submitted to liver transplantation: assessment of efficacy at explants analysis and of safety for tumor recurrence. Liver Transpl 2005; 11: 1117-26.

[28] Brillet PY, Paradis V, Brancatelli G, et al. Percutaneous radiofrequency ablation for hepatocellular carcinoma before liver transplantation: a prospective study with histopathologic comparison. AJR Am J Roentgenol 2006; 186: S296-305.

[29] Rodriguez - Sanjuàn JC, Gonzalez F, Juanco C, et al. Radiological and pathological assessment of hepatocellular carcinoma response to radiofrequency ablation. A study on removed liver after transplantation. World J Surg 2008; 32: 1489-94.

[30] Porrett PM, Peterman H, Rosen M, et al. Lack of benefit of pretransplant locoregional hepatic therapy for hepatocellular cancer in the current MELD era. Liver Transpl 2006; 12: 665-73.

[31] Castroagudin JF, Delgado M, Villanueva A, et al. Safety of percutaneous ethanol injection as neoadjuvant therapy for hepatocellular carcinoma in waiting list liver transplant candidates. Transplant Proc 2005; 37: 3871-3.

[32] Pacella CM, Bizzarri G, Francica G, et al. Analysis of factors predicting survival in patients with hepatocellular carcinoma treated with percutaneous laser ablation. J Hepatol 2006; 44: 902-9.

[33] Gough-Palmer AL, Gedroyc WM. Laser ablation of hepatocellular carcinoma - a review. World J Gastroenterol 2008; 14: 7170-4.

[34] Pompili M, Pacella CM, Francica G, et al. Percutaneous laser ablation of hepatocellular carcinoma in patients with liver cirrhosis awaiting liver transplantation. Eur J Radiol 2009 Apr 2. [Epub ahead of print]

[35] Riaz A, Kulik L, Lewandowski RJ, et al. Radiologic-pathologic correlation of hepatocellular carcinoma treated with internal radiation using yttrium-90 microspheres. Hepatology 2009; 49: 1185-93.

[36] Yao FY, Ferrell L, Bass NM, et al. Liver transplantation for hepatocellular carcinoma: expansion of the tumor size limits does not adversely impact survival. Hepatology 2001; 33: 1394-403.

[37] Chapman WC, Majella Doyle MB, Stuart JE, et al. Outcomes of neoadjuvant transarterial chemoembolization to downstage hepatocellular carcinoma before liver transplantation. Ann Surg 2008; 248: 617-25.

[38] Ravaioli M, Grazi GL, Piscaglia F, et al. Liver transplantation for hepatocellular carcinoma: results of down-staging in patients initially outside the Milan selection criteria. Am J Transplant 2008; 8: 2547-57.

[39] Bharat A, Brown DB, Crippin JS, et al. Pre-liver transplantation locoregional adjuvant therapy for hepatocellular carcinoma as a strategy to improve longterm survival. J Am Coll Surg 2006; 203: 411-20.

[40] Lao OB, Weissman J, Perkins JD. Pre-transplant therapy for hepatocellular carcinoma is associated with a lower recurrence after liver transplantation. Clin Transplant 2009. [Epub ahead of print]

[41] Heckman JT, Devera MB, Marsh JW, et al. Bridging locoregional therapy for hepatocellular carcinoma prior to liver transplantation. Ann Surg Oncol 2008; 15: 3169-77. 
[42] Schwartz M, Roayaie S, Uva P. Treatment of HCC in patients awaiting liver transplantation. Am J Transplant 2007; 7: 1875-81.
[43] Bruix J, Sherman M. Management of hepatocellular carcinoma. Practice Guidelines Committee, American Association for the Study of Liver Diseases. Hepatology 2005; 42: 1208-36.

\section{(C) Pompili et al:; Licensee Bentham Open.}

This is an open access article licensed under the terms of the Creative Commons Attribution Non-Commercial License (http://creativecommons.org/ licenses/by-nc/3.0/), which permits unrestricted, non-commercial use, distribution and reproduction in any medium, provided the work is properly cited. 